\title{
Physician warnings over fitness to drive improve road safety
}

Published online: 05 October 2012

(C) Springer Healthcare 2012

medwireNews: Physician warnings to patients who are potentially unfit to drive may lead to substantial reductions in road crash trauma, report Canadian researchers.

But such warnings may also be linked to the exacerbation of mood disorders and worsening doctor-patient relationships, say Donald Redelmeier, from Sunnybrook Health Sciences Centre, in Toronto, Ontario, and co-authors.

Noting that patients often overestimate their driving skills and fail to take protective actions, the team writes in The New England Journal of Medicine: "Our findings thus pose an ethical conflict for clinicians who seek to optimize patients' health yet respect their preferences...

"Consequently, clinical judgment is needed in deciding which patients are most likely to benefit from a warning."

The researchers examined the records of 100,075 patients who received a medical warning for being potentially unfit to drive from 6098 physicians between 2006, when an incentive to provide warnings was introduced, and 2009. During this period, the proportion of all licensed drivers who received warnings increased from $0.46 \%$ to $0.65 \%$.

Over a 3-year baseline interval, patients who subsequently received a warning were involved in 1430 road crashes that resulted in an emergency department (ED) visit, which corresponds to a rate of 4.76 events per 1000 persons per year, and approximately twice the rate in the general population. In the year after the warning, the patients were involved in 273 crashes that resulted in an ED visit, representing a significant relative risk reduction of $45 \%$.

The relative risk reduction was observed in all patient subgroups, and was increased among patients with at least five of the 20 most common diagnoses, at an annual risk reduction of $52 \%$.

However, the number of ED visits for depression increased from 19.15 events per 1000 patients annually during the baseline period to 23.91 events per 1000 patients during follow up. In addition, the number of patient visits per year to the physician who gave the warning decreased, from 281,971 in the year before to 215,973 in the year after the warning, with a reduction in visits recorded for $29 \%$ of patients.

By Liam Davenport, medwireNews Reporter

\section{Reference}

N Engl J Med 2012; 367: 1228-1236 